The Journal of Nonlinear $\mathbf{S}$ ciences and Applications http://www.tjnsa.com

\title{
ON 2-STRONG HOMOMORPHISMS AND 2-NORMED HYPERSETS IN HYPERVECTOR SPACES
}

\author{
P. RAJA ${ }^{1}$ AND S. M. VAEZPOUR ${ }^{2, *}$
}

\begin{abstract}
In this paper the notion of a 2-normed hyperset in hypervector spaces is introduced. Also we construct some special 2-normed hypersets of strong homomorphisms over hypervector spaces. Among other results we consider 2-strong homomorphisms and investigate some of their properties.
\end{abstract}

\section{INTRODUCTION AND PRELIMINARIES}

In 1964, Gähler introduced the definition of a 2-norm space, see [1]. Subsequently, many authors worked on this new construction and proved many theorems about it, see for example [5], [6], and [11]. In 1999, Lewandowska gave a generalization of Gähler's 2-norm in [2] as following:

Let $X$ and $Y$ be real linear spaces and $\mathcal{D}$ a non-empty subset of $X \times Y$ such that for every $x \in X$ and $y \in Y$ the sets

$$
\mathcal{D}_{x}=\{y \in Y:(x, y) \in \mathcal{D}\} \quad \text { and } \mathcal{D}^{y}=\{x \in X:(x, y) \in \mathcal{D}\}
$$

are linear subspaces of the space $Y$ and $X$, respectively.

A function $\|.,\|:. \mathcal{D} \longrightarrow[0, \infty)$ will be called a generalized $2-$ norm on $\mathcal{D}$ if it satisfies the following conditions:

(i) $\|x, \alpha y\|=|\alpha|\|x, y\|=\|\alpha x, y\|$, for any real number $\alpha$ and all $(x, y) \in \mathcal{D}$,

(ii) $\|x, y+z\| \leqslant\|x, y\|+\|x, z\|$, for $x \in X, y, z \in Y$ such that $(x, y),(x, z) \in$ $\mathcal{D}$

(iii) $\|x+y, z\| \leqslant\|x, y\|+\|w, y\|$, for $x, y \in X, y, z \in Y$ such that $(x, z),(y, z) \in$ $\mathcal{D}$.

Date: Received: 13 May 2008; Revised: 14 Dec 2008.

* Corresponding author.

2000 Mathematics Subject Classification. Primary 46A32; Secondary 46A99.

Key words and phrases. 2-normed set, 2-normed space, Hypervector space, Strong homomorphism. 
Some of mathematicians worked on this construction and found some generalizations of classic theorems. In 1988, Scafati Tallini defined the concept of hypervector spaces and recently, we have proved some theorems about them, see [7], [8], and [9].

Let $P(X)$ be the power set of a set $X, P^{\star}(X)=P(X) \backslash\{\varnothing\}$, and $K$ a field. A hypervector space over $K$ that is defined in [7], is a quadruplet $(X,+, \circ, K)$ such that $(X,+)$ is an abelean group and

$$
\circ: K \times X \longrightarrow P^{\star}(X)
$$

is a mapping that for all $a, b \in K$ and $x, y \in X$ the following properties holds:

(i) $(a+b) \circ x \subseteq(a \circ x)+(b \circ x)$,

(ii) $a \circ(x+y) \subseteq(a \circ x)+(a \circ y)$,

(iii) $a \circ(b \circ x)=(a b) \circ x$, where $a \circ(b \circ x)=\{a \circ y: y \in b \circ x\}$,

(iv) $(-a) \circ x=a \circ(-x)$,

(v) $x \in 1 \circ x$.

A non-empty subset of a hypervector space $X$ over a field $K$ is called a subspace of $X$ if the following hold:

(i) $H-H \subseteq H$,

(ii) $a \circ H \subseteq H$, for every $a \in K$.

Let $(X,+, \circ, K)$ be a hypervector space. Suppose that for every $a \in K,|a|$ denoted the valuation of $a$ in $K$. A norm on a hypervector space, $X$, is a mapping

$$
\|\cdot\|: X \longrightarrow \mathbb{R}
$$

that for all $a \in K$ and $x, y \in X$ has the following properties:

(i) $\|x\|=0$ if and only if $x=0$,

(ii) $\|x+y\| \leqslant\|x\|+\|y\|$,

(iii) $\sup \|a \circ x\|=|a|\|x\|$,

Let $\left(X,+_{1}, \circ_{1}, K\right)$ and $\left(Y,+_{2}, o_{2}, K\right)$ be two hypervector spaces. A strong homomorphism between $X$ and $Y$, is a mapping

$$
f: X \longrightarrow Y
$$

such that for all $a \in K$ and $x, y \in X$ the following hold:

(i) $f\left(x+{ }_{1} y\right)=f(x)+{ }_{2} f(y)$,

(ii) $f\left(a \circ_{1} x\right)=a \circ_{2} f(x)$.

For more details see [7], [8], and [10].

Remark 1.1. Let $X=\left(X,+_{1}, \circ_{1}, \mathbb{R}\right)$ and $Y=\left(Y,+_{2}, o_{2}, \mathbb{R}\right)$ be real hypervector spaces. Assume that $L(X, Y)$ denotes the set of all strong homomorphisms from $X$ to $Y$. For every $f, g \in L(X, Y), \alpha \in \mathbb{R}$, and $x \in X$, suppose that:

(i) $(f+g)(x)=f(x)+{ }_{2} g(x)$,

(ii) $\alpha \circ f=\left\{h \in L(X, Y): h(x) \in f\left(\alpha \circ \circ_{1} x\right)\right.$, for every $\left.x \in X\right\}$.

It is not hard to see that $(L(X, Y),+, \circ, \mathbb{R})$ is a real hypervector space. 
In this paper the notion of a 2-normed hyperset in hypervector spaces is introduced. Also we construct some special 2-normed hypersets of strong homomorphisms over hypervector spaces. Among other results we consider 2-strong homomorphisms and investigate some of their properties.

\section{MAin REsults}

Let $X=\left(X,+_{1}, \circ_{1}, \mathbb{R}\right)$ and $Y=\left(Y,+_{2}, \circ_{2}, \mathbb{R}\right)$ be real hypervector spaces, and $\mathcal{D}$ a non-empty subset of $X \times Y$. Put $\mathcal{D}_{x}=\{y \in Y:(x, y) \in \mathcal{D}\}$ and $\mathcal{D}^{y}=\{x \in X:(x, y) \in \mathcal{D}\}$, for every $x \in X$ and $y \in Y$.

Definition 2.1. Let $X=\left(X,+_{1}, \circ_{1}, \mathbb{R}\right)$ and $Y=\left(Y,+_{2}, \circ_{2}, \mathbb{R}\right)$ be real hypervector spaces. Let $\mathcal{D}$ be a non-empty subset of $X \times Y$.

A function $\|.\|:, \mathcal{D} \longrightarrow[0, \infty)$ is called a generalized $2-$ norm on $\mathcal{D}$ if for all $x, w \in X, y, z \in Y$ such that $(x, y),(x, z),(w, y) \in \mathcal{D}$, and $\alpha \in \mathbb{R}$, the following hold:

(i) $\sup \left\|x, \alpha \circ_{2} y\right\|=|\alpha|\|x, y\|=\sup \left\|\alpha \circ_{1} x, y\right\|$,

(ii) $\left\|x, y+{ }_{2} z\right\| \leqslant\|x, y\|+\|x, z\|$,

(iii) $\left\|x+{ }_{1} w, y\right\| \leqslant\|x, y\|+\|w, y\|$.

Moreover if $\mathcal{D}_{x}$ and $\mathcal{D}^{y}$ are subspaces of the hypervector spaces $Y$ and $X$, respectively, for every $x \in X, y \in Y$, then the set $\mathcal{D}$ is called a 2 -normed hyperset. In particular, if $\mathcal{D}=X \times Y$, the function $\| .$, .|| is called a generalized 2-norm on $X \times Y$ and the pair $(X \times Y,\|.,\|$.$) a generalized 2$-normed hypervector space. Also if $X=Y$, then the generalized 2-normed hypervector space is denoted by $(X,\|.,\|$.$) .$

Definition 2.2. Let $X=(X,+, \circ, \mathbb{R})$ be real hypervector space. Let $\mathcal{D}$ be a nonempty subset of $X \times X$ such that $\mathcal{D}=\mathcal{D}^{-1}$, where $\mathcal{D}^{-1}=\{(y, x):(x, y) \in \mathcal{D}\}$, and the set $\mathcal{D}^{y}$ is a subspace of $X$, for every $y \in X$.

A generalized symmetric $2-$ norm on $\mathcal{D}$ is a generalized 2 -norm on $\mathcal{D}$ such that $\|x, y\|=\|y, x\|$, for every $(x, y) \in \mathcal{D}$.

The set $\mathcal{D}$ is called a generalized symmetric 2 -normed hyperset. Specially, if $\mathcal{D}=X \times X$, the function $\|.,$.$\| is called a generalized symmetric 2$-norm on $X$ and the pair $(X,\|.,\|$.$) a generalized symmetric 2$-normed hypervector space.

Example 2.3. Let $S$ be the set of all sequences of real numbers. For every $\left\{r_{n}\right\},\left\{s_{n}\right\} \in S$ and $\alpha \in \mathbb{R}$, define:

(i) $\left\{r_{n}\right\}+\left\{s_{n}\right\}=\left\{r_{n}+s_{n}\right\}$,

(ii) $\alpha \circ\left\{s_{n}\right\}=\left\{\left\{t_{n}\right\}: t_{n}\right.$ is in the segment between zero and $\alpha s_{n}$, for $n \in$ $\mathbb{N}\}$.

It is easily check that $(S,+, \circ, \mathbb{R})$ is a hypervector space. Let

$$
\left\|\left\{s_{n}\right\},\left\{r_{n}\right\}\right\|=\sum_{n=1}^{\infty}\left|s_{n}\right|\left|r_{n}\right|
$$

for all $\left\{r_{n}\right\},\left\{s_{n}\right\} \in S$. If $\mathcal{D}=\left\{\left(\left\{r_{n}\right\},\left\{s_{n}\right\}\right) \in S^{2}:\left\|\left\{r_{n}\right\},\left\{s_{n}\right\}\right\|<\infty\right\}$, then we have:

(i) $\mathcal{D}=\mathcal{D}^{-1}$, 
(ii) $\mathcal{D}^{\left\{s_{n}\right\}}$ is a subspace of $S$ for every $\left\{s_{n}\right\} \in S$.

So $\|.,\|:. \mathcal{D} \longrightarrow[0, \infty)$ is a generalized symmetric $2-$ norm on $\mathcal{D}$, and $\mathcal{D}$ is a symmetric 2 -normed hyperset.

In this section we will consider bounded 2-strong homomorphisms defined on a 2-normed hyperset into a normed hypervector space.

Definition 2.4. Let $X=\left(X,+_{1}, \circ_{1}, \mathbb{R}\right)$ be a hypervector space, $\mathcal{D} \subseteq X \times X$ a 2-normed hyperset, and $Y=\left(Y,+_{2}, \circ_{2},\|\|,. \mathbb{R}\right)$ be a normed hypervector space. An operator $F: \mathcal{D} \longrightarrow Y$ is said to be a 2 -strong homomorphism if the following hold:

(i) $F(a+c, b+d)=F(a, b)+F(a, d)+F(c, b)+F(c, d)$, for $a, b, c, d \in X$ such that $a, c \in \mathcal{D}^{b} \cap \mathcal{D}^{d}$,

(ii) $F\left(\alpha \circ_{1} a, \beta \circ_{1} b\right)=(\alpha \beta) \circ_{2} F(a, b)$, for $\alpha, \beta \in \mathbb{R},(a, b) \in \mathcal{D}$.

A 2 -strong homomorphism is called bounded, if there is $M>0$ such that

$$
\|F(a, b)\| \leqslant M\|\| a, b \|,
$$

for every $(a, b) \in \mathcal{D}$. In this case we define

$$
\|F\|=\inf \{M>0: \forall(a, b) \in \mathcal{D}\|F(a, b)\| \leqslant M\|\mid a, b\|\}
$$

as the norm of the $F$.

Theorem 2.5. Let $X=\left(X,+_{1}, \circ_{1}, \mathbb{R}\right)$ and $Y=\left(Y,+_{2}, \circ_{2},\|\|,. \mathbb{R}\right)$ be hypervector spaces, and $\mathcal{D} \subseteq X \times X$ a 2 -normed hyperset. If $F: \mathcal{D} \longrightarrow Y$ is a bounded 2-strong homomorphism, then we have the following:

(i) $\|F\| \leqslant M$, for $M \in \mathcal{P}^{(F)}$, where $\mathcal{P}^{(F)}=\{K>0:\|F(a, b)\| \leqslant$ $K\|a, b\|$ for all $(a, b) \in \mathcal{D}\}$,

(ii) $\|F(a, b)\| \leqslant\|F\|\|a, b\|$, for every $(a, b) \in \mathcal{D}$,

$$
\begin{aligned}
\|F\| & =\sup \{\|F(a, b)\|:(a, b) \in \mathcal{D},\|a, b\| \leqslant 1\} \\
& =\sup \left\{\frac{\|F(a, b)\|}{\|a, b\|}:(a, b) \in \mathcal{D},\|a, b\| \neq 0\right\} .
\end{aligned}
$$

Proof. The proofs of (i) and (ii) are obvious.

To prove (iii), by condition (ii), we have

$$
\sup \left\{\frac{\|F(a, b)\|}{\|(a, b)\|}:(a, b) \in \mathcal{D},\|a, b\| \neq 0\right\} \leqslant\|F\| .
$$

Let $\alpha=\sup \{\|F(a, b)\|:(a, b) \in \mathcal{D},\|a, b\| \leqslant 1\}$. Then

$$
\begin{aligned}
\alpha & =\sup \{\|F(a, b)\|:(a, b) \in \mathcal{D},\|a, b\| \leqslant 1\} \\
& \leqslant \sup \left\{\frac{\|F(a, b)\|}{\|a, b\|}:(a, b) \in \mathcal{D},\|a, b\| \leqslant 1\right\} \\
& \leqslant \sup \left\{\frac{\|F(a, b)\|}{\|a, b\|}:(a, b) \in \mathcal{D},\|a, b\| \neq 0\right\} \\
& \leqslant\|F\| .
\end{aligned}
$$


Let $(a, b) \in \mathcal{D}$ be such that $\|a, b\| \neq 0$. Since $\sup \left\|\frac{1}{\|a, b\|} \circ_{1} a, b\right\|=\frac{1}{\|a, b\| \|}\|a, b\|=$ 1 , then we have $\|c, d\| \leqslant 1$, for every $(c, d) \in\left(\frac{1}{\|a, b\|} \circ_{1} a, b\right)$, and so

$$
\frac{1}{\|a, b\|}\|F(a, b)\|=\sup \left\|\frac{1}{\|a, b\|} \circ_{2} F(a, b)\right\|=\sup \left\|F\left(\frac{1}{\|a, b\|} \circ_{1} a, b\right)\right\| \leqslant \alpha .
$$

On the other hand if $(a, b) \in \mathcal{D}$ and $\|a, b\|=0$, then $0 \leqslant\|F(a, b)\| \leqslant$ $\|F \mid\|\|a, b\|=0$ and so $\|F(a, b)\|=0$. Hence $\|F(a, b)\| \leqslant \alpha\|a, b\|$, for every $(a, b) \in \mathcal{D}$, and therefore $\alpha \in \mathcal{P}^{(F)}$. So $\|F\| \leqslant \alpha$. This completes the proof.

Remark 2.6. Let $X=\left(X,+_{1}, \circ_{1}, \mathbb{R}\right)$ and $Y=\left(Y,+_{2}, \circ_{2}, \mathbb{R}\right)$ be hypervector spaces, and $\mathcal{D} \subseteq X \times X$ a 2 -normed hyperset. Denote by $L_{2}(D, Y)$ the set of all bounded 2-strong homomorphisms from $\mathcal{D}$ into $Y$. For every $F, G \in L_{2}(D, Y)$, $\alpha \in \mathbb{R}$, and $(a, b) \in \mathcal{D}$, suppose that:

(i) $(F+G)(a, b)=F(a, b)+{ }_{2} G(a, b)$,

(ii) $\alpha \circ F=\left\{H \in L_{2}(D, Y): H(a, b) \in \alpha \circ_{2} F(a, b)\right.$, for every $\left.(a, b) \in \mathcal{D}\right\}$.

It is not hard to see that $\left(L_{2}(D, Y),+, \circ, \mathbb{R}\right)$ is a real hypervector space.

Theorem 2.7. Let $X=\left(X,+_{1}, \circ_{1}, \mathbb{R}\right)$ and $Y=\left(Y,+_{2}, \circ_{2},\|\|,. \mathbb{R}\right)$ be hypervector spaces, and $\mathcal{D} \subseteq X \times X$ a 2 -normed hyperset. If we define

$$
\|F\|=\inf \{M>0: \forall(a, b) \in \mathcal{D}\|F(a, b)\| \leqslant M\|\mid a, b\|\},
$$

for every $F \in L_{2}(D, Y)$, then $\left(L_{2}(D, Y),+, \circ,\|\|,. \mathbb{R}\right)$ is a normed hypervector space.

Proof. If $\|F\|=0$, then $\|F(a, b)\|=0$, for every $(a, b) \in \mathcal{D}$. Thus $F(a, b)=0$, for every $(a, b) \in \mathcal{D}$. Conversely, if $F$ is the zero 2 -strong homomorphism, then

$$
\|F\|=\sup \{\|F(a, b)\|:(a, b) \in \mathcal{D},\|a, b\| \leqslant 1\}=0 .
$$

Now, suppose that $F, G \in L_{2}(D, Y)$. We have

$$
\begin{aligned}
\|(F+G)(a, b)\| & =\|F(a, b)+G(a, b)\| \\
& \leqslant\|F(a, b)\|+\|G(a, b)\| \\
& \leqslant\|F\|\|\mid a, b\|+\|G\|\|a, b\| \\
& =(\|F\|+\|G\|)\|a, b\|,
\end{aligned}
$$

for every $(a, b) \in \mathcal{D}$. So $\|F+G\| \leqslant\|F\|+\|G\|$.

Finally, suppose that $\alpha \in \mathbb{R}$. We have

$$
\sup \|(\alpha \circ F)(a, b)\| \leqslant \sup \left\|\alpha \circ_{2} F(a, b)\right\| \leqslant|\alpha|\|F(a, b)\| \leqslant|\alpha|\|F\|\|a, b\|,
$$

for every $(a, b) \in \mathcal{D}$. So $\sup \|\alpha \circ F\|=|\alpha|\|F\|$. Now, assume that $\alpha \neq 0$, then

$$
\|F\| \leqslant \sup \left\|\frac{1}{\alpha} \circ(\alpha \circ F)\right\| \leqslant \sup \mid \frac{1}{\alpha}\|\alpha \circ F\| .
$$

Hence $|\alpha|\|F\| \leqslant \sup \|\alpha \circ F\|$, and therefore $\sup \|\alpha \circ F\|=|\alpha\||| F\|$, for every $\alpha \neq 0$. On the other hand it is obvious that this equality is true for $\alpha=0$. So the proof is complete.

We recall that if $X=(X,+, \circ, \mathbb{R})$ is a real hypervector space, then $(X \times$ $X,+, \circ, \mathbb{R})$ is also a hypervector space with the following operations: 
(i) $x+y=\left(x_{1}+{ }_{1} y_{1}, x_{2}+{ }_{1} y_{2}\right)$,

(ii) $\alpha \circ\left(x_{1}, x_{2}\right)=\left(\alpha \circ_{1} x_{1}, \alpha \circ_{1} x_{2}\right)$, for $x=\left(x_{1}, x_{2}\right), y=\left(y_{1}, y_{2}\right) \in X \times X$ and $\alpha \in \mathbb{R}$.

Let $X$ and $Y$ be hypervector spaces. Clearly, if $F: X \longrightarrow Y \times Y$ is a strong homomorphism, then there are $f, g \in L(X, Y)$ such that $F(x)=(f(x), g(x))$, for every $x \in X$. Also the function $F: X \longrightarrow Y \times Y$ defined by the formula $F(x)=$ $(f(x), g(x))$, for every $x \in X$, where $f, g \in L(X, Y)$, is a strong homomorphism.

Now, we are going to construct two 2-normed hypersets of $L(X, Y)^{2}$.

Definition 2.8. Let $X=\left(X,+_{1}, \circ_{1},\|.\|_{1}, \mathbb{R}\right)$ be a real normed hypervector space, $Y$ a real hypervector space, and $\mathcal{Y} \subseteq Y \times Y$ a 2 -normed hyperset. The set $\mathcal{M}_{\mathcal{Y}} \subseteq L(X, Y)^{2}$ is a set that $(f, g) \in \mathcal{M}_{\mathcal{Y}}$ if for every $x \in X$, the following hold:

(i) $(f(x), g(x)) \in \mathcal{Y}$,

(ii) There is $M>0$ such that $\|f(x), g(x)\| \leqslant M\|x\|_{1}^{2}$.

Lemma 2.9. Let $X=\left(X,+_{1}, \circ_{1},\|.\|_{1}, \mathbb{R}\right)$ and $Y=\left(Y,+_{2}, \circ_{2}, \mathbb{R}\right)$ be hypervector spaces. The set $\mathcal{M}_{\mathcal{Y}}$, for $\mathcal{Y} \subseteq Y \times Y$ has the following properties:

(i) If $\mathcal{Y}$ is a symmetric 2-normed hyperset, then $\mathcal{M}_{\mathcal{Y}}=\mathcal{M}_{\mathcal{Y}}^{-1}$,

(ii) The sets $\mathcal{M}_{\mathcal{Y}}^{g}=\left\{h \in L(X, Y):(h, g) \in \mathcal{M}_{\mathcal{Y}}\right\}$ and $\left(\mathcal{M}_{\mathcal{Y}}\right)_{f}=\{h \in$ $\left.L(X, Y):(f, h) \in \mathcal{M}_{\mathcal{Y}}\right\}$ are subspaces of the hypervector space $L(X, Y)$, for every $f, g \in L(X, Y)$.

Moreover, if $\mathcal{Y}$ is a symmetric 2-normed hyperset, we have $\left(\mathcal{M}_{\mathcal{Y}}\right)_{f}=\mathcal{M}_{\mathcal{Y}}^{f}$.

Proof. Clearly, (i) is a consequence of the definition of $\mathcal{M}_{\mathcal{Y}}$.

To prove (ii), let $g: X \longrightarrow Y$ be a strong homomorphism. Assume that $h: X \longrightarrow Y$ is a strong homomorphism such that $h(x)=0$, for every $x \in X$. We show that if $H$ is a subspace of $Y$, then $0 \in H$. Since $H$ is a subspace, then $H \neq \varnothing$, so suppose that $y \in H$. Now, we have $-y \in(-1) \circ_{2} y$ and therefore $0=y-y \in H+(-1) \circ_{2} y \subseteq H+H \subseteq H$. Since for every $x \in X$, the set $\mathcal{Y}^{g(x)}$ is a subspace of $Y$, then $h(x)=0 \in \mathcal{Y}^{g(x)}$, for every $x \in X$. Therefore $(h(x), g(x)) \in \mathcal{Y}$, for every $x \in X$. Also if $M>0$, then

$$
\|h(x), g(x)\|=\|0, g(x)\|=0 \leqslant M\|x\|_{1}^{2},
$$

for every $x \in X$. So $H \in \mathcal{M}_{\mathcal{Y}}^{g}$ and $\mathcal{M}_{\mathcal{Y}}^{g} \neq \varnothing$.

Now, let $f_{1}, f_{2} \in \mathcal{M}_{\mathcal{Y}}^{g}$. Therefore $\left(f_{1}(x), g(x)\right),\left(f_{2}(x), g(x)\right) \in \mathcal{Y}$ and so $f_{1}(x), f_{2}(x) \in \mathcal{Y}^{g(x)}$. Since $\mathcal{Y}^{g(x)}$ is a subspace of $Y$, then $f_{1}(x)+f_{2}(x) \in \mathcal{Y}^{g(x)}$, and we have $\left(f_{1}+f_{2}\right)(x, g(x)) \in \mathcal{Y}$, for every $x \in X$. Moreover there are $M_{1}, M_{2}>0$ such that $\left\|f_{1}(x), g(x)\right\| \leqslant M_{1}\|x\|_{1}^{2}$ and $\left\|f_{2}(x), g(x)\right\| \leqslant M_{2}\|x\|_{1}^{2}$, for every $x \in X$. Hence

$$
\left\|f_{1}(x)+f_{2}(x), g(x)\right\| \leqslant\left\|f_{1}(x), g(x)\right\|+\left\|f_{2}(x), g(x)\right\| \leqslant\left(M_{1}+M_{2}\right)\|x\|_{1}^{2},
$$

for every $x \in X$. Therefore $f_{1}+f_{2} \in \mathcal{M}_{\mathcal{Y}}^{g}$.

Suppose that $\alpha \in \mathbb{R}, f \in \mathcal{M}_{\mathcal{Y}}^{g}$. It shows that $(f(x), g(x)) \in \mathcal{Y}$ and consequently, $f(x) \in \mathcal{Y}^{g(x)}$, for every $x \in X$. Since $\mathcal{Y}^{g(x)}$ is a subspace of $Y$, then 
$\alpha \circ_{2} f(x) \subseteq \mathcal{Y}^{g(x)}$ and therefore

$$
\left.\left(\alpha \circ_{2} f(x), g(x)\right)=\left\{(t, g(x)): t \in \alpha \circ_{2} f(x)=f\left(\alpha \circ_{1} x\right)\right)\right\} \subseteq \mathcal{Y},
$$

for every $x \in X$. Moreover, there exists $M>0$ such that $\|f(x), g(x)\| \leqslant M\|x\|_{1}^{2}$, for every $x \in X$. Hence for $h \in \alpha \circ f$, we have

$$
\|h(x), g(x)\| \leqslant \sup \left\|\alpha \circ_{2} f(x), g(x)\right\|=|\alpha|\|f(x), g(x)\| \leqslant|\alpha| M\|x\|_{1}^{2},
$$

for every $x \in X$. Therefore $\alpha \circ f \subseteq \mathcal{M}_{\mathcal{Y}}^{g}$. So $\mathcal{M}_{\mathcal{Y}}^{g}$ is a subspace of $L(X, Y)$.

Similarly, it can be shown that $\left(\mathcal{M}_{\mathcal{Y}}\right)_{f}$ is a subspace of $L(X, Y)$. The condition (i) implies the equality $\left(\mathcal{M}_{\mathcal{Y}}\right)_{f}=\mathcal{M}_{\mathcal{Y}}^{f}$. This completes the proof.

Definition 2.10. Let $X=\left(X,+_{1}, \circ_{1},\|.\|_{1}, \mathbb{R}\right)$ and $Y=\left(Y,+_{2}, \circ_{2}, \mathbb{R}\right)$ be hypervector spaces and $(f, g) \in \mathcal{M}_{\mathcal{Y}}$, for $\mathcal{Y} \subseteq Y \times Y$. Define

$$
\|f, g\|=\inf \left\{M>0:\|f(x), g(x)\| \leqslant M\|x\|_{1}^{2} \text { for all } x \in X\right\} .
$$

Theorem 2.11. Let $X=\left(X,+_{1}, \circ_{1},\|.\|_{1}, \mathbb{R}\right)$ and $Y=\left(Y,+_{2}, \circ_{2}, \mathbb{R}\right)$ be hypervector spaces and $(f, g) \in \mathcal{M}$, for $\mathcal{Y} \subseteq Y \times Y$. Then the following hold:

(i) $\|f, g\| \leqslant M$, for every $M \in P^{(f, g)}$, where

$$
P^{(f, g)}=\left\{K>0:\|f(x), g(x)\| \leqslant K\|x\|_{1}^{2} \text { for all } x \in X\right\},
$$

(ii) $\|f(x), g(x)\| \leqslant\|f, g\|\|x\|_{1}^{2}$, for every $x \in X$,

(iii)

$$
\begin{aligned}
\|f, g\| & =\sup \left\{\|f(x), g(x)\|: x \in X,\|x\|_{1} \leqslant 1\right\} \\
& =\sup \left\{\frac{\|f(x), g(x)\|}{\|x\|_{1}^{2}}: x \in X,\|x\|_{1} \neq 0\right\} .
\end{aligned}
$$

(iv) If $\mathcal{Y}$ is a symmetric 2-normed hyperset, then $\|f, g\|=\|g, f\|$, for every $(f, g) \in \mathcal{M}_{\mathcal{Y}}$.

Proof. The proofs of (i), (ii), and (iv) are obvious.

To prove (iii), by condition (ii), we have

$$
\sup \left\{\|f(x), g(x)\|: x \in X,\|x\|_{1} \leqslant 1\right\} \leqslant\|f, g\| .
$$

Let $\alpha=\sup \left\{\|f(x), g(x)\|: x \in X,\|x\|_{1} \leqslant 1\right\}$, and $x \in X$ with $x \neq 0$. Then

$$
\begin{aligned}
\|f(x), g(x)\| & =\sup \left\|\left(\frac{1}{\|x\|_{1}}\|x\|_{1}\right) \circ_{2} f(x),\left(\frac{1}{\|x\|_{1}}\|x\|_{1}\right) \circ_{2} g(x)\right\| \\
& =\sup \|\| x\left\|_{1} \circ_{2} f\left(\frac{1}{\|x\|_{1}} \circ_{1} x\right),\right\| x\left\|_{1} \circ_{2} g\left(\frac{1}{\|x\|_{1}} \circ_{1} x\right)\right\| \\
& =\|x\|_{1}^{2} \sup \left\|f\left(\frac{1}{\|x\|_{1}} \circ_{1} x\right), g\left(\frac{1}{\|x\|_{1}} \circ_{1} x\right)\right\| .
\end{aligned}
$$

If $y \in \frac{1}{\|x\|_{1}} \circ_{1} x$, then $\|y\| \leqslant 1$ and $\|f(y), g(y)\| \leqslant \alpha$. Thus $\|f(x), g(x)\| \leqslant \alpha\|x\|_{1}^{2}$, for $x \neq 0$. Also $\|f(x), g(x)\|=0 \leqslant \alpha\|x\|_{1}^{2}$, for $x=0$. Therefore $\|f(x), g(x)\| \leqslant$ $\alpha\|x\|_{1}^{2}$, for every $x \in X$, and it means that $\alpha \in P^{(f, g)}$. By (i), we have $\|f, g\| \leqslant \alpha$ and by (1), we conclude that $\|f, g\|=\sup \left\{\|f(x), g(x)\|: x \in X,\|x\|_{1} \leqslant 1\right\}$. 
Now, suppose that $0 \neq x \in X$. By (ii), we have

$$
\frac{\|f(x), g(x)\|}{\|x\|_{1}^{2}} \leqslant\|f, g\|,
$$

and so

$$
\sup \left\{\frac{\|f(x), g(x)\|}{\|x\|_{1}^{2}}: x \in X,\|x\|_{1} \neq 0\right\} \leqslant\|f, g\| .
$$

So if we put $\beta=\sup \left\{\frac{\|f(x), g(x)\|}{\|x\|_{1}^{2}}: x \in X,\|x\|_{1} \neq 0\right\}$, then $\|f(x), g(x)\| \leqslant \beta\|x\|_{1}^{2}$, for every $x \neq 0$. On the other hand if $x=0$, then $\|f(x), g(x)\|=0$. Thus $\|f(x), g(x)\| \leqslant \beta\|x\|_{1}^{2}$, for every $x \in X$, which means that $\beta \in P^{(f, g)}$ and $\|f, g\| \leqslant \beta$. This completes the proof.

Theorem 2.12. Let $X=\left(X,+_{1}, \circ_{1},\|.\|_{1}, \mathbb{R}\right)$ and $Y=\left(Y,+_{2}, \circ_{2}, \mathbb{R}\right)$ be hypervector spaces. The set $\mathcal{M}_{\mathcal{Y}}$, for $\mathcal{Y} \subseteq Y \times Y$, is a 2-normed hyperset with the following 2-norm:

$$
\|f, g\|=\sup \left\{\|f(x), g(x)\|: x \in X,\|x\|_{1} \leqslant 1\right\},
$$

for $(f, g) \in \mathcal{M}_{\mathcal{Y}}$. Moreover, if $\mathcal{Y}$ is a symmetric 2 -normed set, then the set $\mathcal{M}_{\mathcal{Y}}$ is also symmetric.

Proof. Suppose that $(f, g) \in \mathcal{M}_{\mathcal{Y}}$. It means that there is $M>0$ such that $\|f(x), g(x)\| \leqslant M\|x\|_{1}^{2}$, for every $x \in X$. Therefore

$$
\sup \left\{\|f(x), g(x)\|: x \in X,\|x\|_{1} \leqslant 1\right\} \leqslant M<\infty,
$$

and so the function ||$.$, . || has finite non-negative values. Moreover we have the following:

(i) If $x \in X,\|x\| \leqslant 1, \alpha \in \mathbb{R}$. Then

$$
\begin{aligned}
\sup \|f(x),(\alpha \circ g)(x)\| & \leqslant \sup \|f(x), \alpha \circ g(x)\| \\
& =|\alpha|\|f(x), g(x)\| \\
& \leqslant|\alpha| \sup \left\{\|f(x), g(x)\|: x \in X,\|x\|_{1} \leqslant 1\right\} \\
& =|\alpha|\|f, g\|,
\end{aligned}
$$

and consequently, we have

$$
\sup \|f, \alpha \circ g\| \leqslant|\alpha|\|f, g\| .
$$

Now, let $\alpha \neq 0$. Using (2), we have

$$
\|f, g\| \leqslant \sup \left\|f,\left(\frac{1}{\alpha} \alpha\right) \circ g\right\| \leqslant \frac{1}{\alpha} \sup \|f, \alpha \circ g\|,
$$

and $|\alpha|\|f, g\| \leqslant \sup \|f, \alpha \circ g\|$, for $\alpha \neq 0$. On the other hand if $\alpha=0$, then $|\alpha|\|f, g\|=0=\sup \|f, \alpha \circ g\|$. So $\mid \alpha\|\| f, g\|\leqslant \sup \| f, \alpha \circ g \|$, for all $\alpha \in \mathbb{R}$, and by using (2), we have

$$
\sup \|f, \alpha \circ g\|=\mid \alpha\|\| f, g \| \text {. }
$$

Similarly, we can show that $\sup \|\alpha \circ f, g\|=|\alpha|\|f, g\|$. 
(ii) Suppose that $f, g, h \in L(X, Y)$ such that $(f, g),(f, h) \in \mathcal{M}_{\mathcal{Y}}$. Let $x \in X$ be such that $\|x\| \leqslant 1$. Then we have

$$
\begin{aligned}
\|f(x),(g+h)(x)\| & =\|f(x), g(x)+h(x)\| \leqslant\|f(x), g(x)\|+\|f(x), h(x)\| \\
& \leqslant \sup \left\{\|f(x), g(x)\|: x \in X,\|x\|_{1} \leqslant 1\right\} \\
& +\sup \left\{\|f(x), h(x)\|: x \in X,\|x\|_{1} \leqslant 1\right\} \\
& =\|f, g\|+\|f, h\| .
\end{aligned}
$$

It implies that

$$
\|f, g+h\| \leqslant\|f, g\|+\|f, h\| .
$$

(iii) In a similar way we may prove that

$$
\|f+g, h\| \leqslant\|f, h\|+\|g, h\| .
$$

Now, suppose that $\mathcal{Y}$ is a symmetric 2 -normed hyperset. Then $\mathcal{M}_{\mathcal{Y}}=\mathcal{M}_{\mathcal{Y}}^{-1}$ and we have

$$
\begin{aligned}
\|f, g\| & =\sup \left\{\|f(x), g(x)\|: x \in X,\|x\|_{1} \leqslant 1\right\} \\
& =\sup \left\{\|g(x), f(x)\|: x \in X,\|x\|_{1} \leqslant 1\right\}=\|g, f\| .
\end{aligned}
$$

Therefore $\|.,$.$\| is a generalized 2-$ norm on $\mathcal{M}_{\mathcal{Y}}$. Moreover by Lemma 2.9, the set $\left(\mathcal{M}_{\mathcal{Y}}\right)_{f}$ and $\left(\mathcal{M}_{\mathcal{Y}}\right)^{f}$ are subspaces of $L(X, Y)$, for every $f \in L(X, Y)$. So the set $\mathcal{M}_{\mathcal{Y}}$ is a symmetric 2 -normed hyperset and this completes the proof of the theorem.

Let $X$ and $Y$ be hypervector spaces, and $f, g \in L(X, Y)$, then the map $(f, g)$ : $X \times X \longrightarrow Y \times Y$ which is defined $(f, g)(x)=(f(x), g(x))$, for every $x \in X$, is a strong homomorphisms as above.

Definition 2.13. Let $X=\left(X,+_{1}, \circ_{1},\|.\|_{1}, \mathbb{R}\right)$ and $Y=\left(Y,+_{2}, \circ_{2},\|.\|_{2}, \mathbb{R}\right)$ be normed hypervector spaces, and $\mathcal{Y} \subseteq Y \times Y$ a 2 -normed hyperset. The set $\mathcal{N}_{\mathcal{Y}} \subseteq L(X, Y)^{2}$ is a set that $(f, g) \in \mathcal{N}_{\mathcal{Y}}$ if for every $x \in X$ and $y \in Y$, the following hold:

(i) $(f(x), g(y)) \in \mathcal{N}_{\mathcal{Y}}$,

(ii) There is $M>0$ such that $\|f(x), g(y)\| \leqslant M\|x\|_{1}\|y\|_{2}$.

Similar to Theorem 2.11 and Theorem 2.12 , we have the following theorems. So their proofs are omitted.

Lemma 2.14. Let $X=\left(X,+_{1}, \circ_{1},\|.\|_{1}, \mathbb{R}\right)$ and $Y=\left(Y,+_{2}, \circ_{2},\|.\|_{2}, \mathbb{R}\right)$ be normed hypervector spaces. The set $\mathcal{N}_{\mathcal{Y}}$, for $\mathcal{Y} \subseteq Y \times Y$ has the following properties:

(i) If $\mathcal{Y}$ is a symmetric $2-$ normed hyperset, then $\mathcal{N}_{\mathcal{Y}}=\mathcal{N}_{\mathcal{Y}}^{-1}$,

(ii) The sets $\mathcal{N}_{\mathcal{Y}}^{g}=\left\{h \in L(X, Y):(h, g) \in \mathcal{N}_{\mathcal{Y}}\right\}$ and $\left(\mathcal{N}_{\mathcal{Y}}\right)_{f}=\{h \in$ $\left.L(X, Y):(f, h) \in \mathcal{N}_{\mathcal{Y}}\right\}$ are subspaces of the hypervector space $L(X, Y)$, for every $f, g \in L(X, Y)$.

Moreover, if $\mathcal{Y}$ is a symmetric 2-normed hyperset, we have $\left(\mathcal{N}_{\mathcal{Y}}\right)_{f}=\mathcal{N}_{\mathcal{Y}}^{f}$.

The proof is similar to the proof of Lemma 2.9, so we omit it. 
Definition 2.15. Let $X=\left(X,+_{1}, \circ_{1},\|.\| \|_{1}, \mathbb{R}\right)$ and $Y=\left(Y,+_{2}, \circ_{2},\|.\|_{2}, \mathbb{R}\right)$ be normed hypervector spaces, and $(f, g) \in \mathcal{N}_{\mathcal{Y}}$, for $\mathcal{Y} \subseteq Y \times Y$. Define

$$
\|f, g\|=\inf \left\{M>0: \forall x \in X, y \in Y\|f(x), g(y)\| \leqslant M\|x\|_{1}\|y\|_{2}\right\} .
$$

Theorem 2.16. Let $X=\left(X,+_{1}, \circ_{1},\|.\| \|_{1}, \mathbb{R}\right)$ and $Y=\left(Y,+_{2}, \circ_{2},\|.\| \|_{2}, \mathbb{R}\right)$ be normed hypervector spaces, and $(f, g) \in \mathcal{N}_{\mathcal{Y}}$, for $\mathcal{Y} \subseteq Y \times Y$. Then the following hold:

(i) $\|f, g\| \leqslant M$, for every $M \in R^{(f, g)}$, where

$R^{(f, g)}=\left\{K>0:\|f(x), g(y)\| \leqslant K\|x\|_{1}\|y\|_{2}\right.$ for every $\left.x \in X, y \in Y\right\}$,

(ii) $\|f(x), g(y)\| \leqslant\|f, g\|\|x\|_{1}\|y\|_{2}$, for every $x \in X$ and $y \in Y$,

(iii)

$$
\begin{aligned}
\|f, g\| & =\sup \left\{\|f(x), g(y)\|: x \in X, y \in Y,\|x\|_{1},\|y\|_{2} \leqslant 1\right\} \\
& =\sup \left\{\frac{\|f(x), g(y)\|}{\|x\|_{1}^{2}}: x \in X, y \in Y,\|x\|_{1},\|y\|_{2} \neq 0\right\} .
\end{aligned}
$$

(iv) If $\mathcal{Y}$ is a symmetric 2-normed hyperset, then $\|f, g\|=\|g, f\|$, for every $(f, g) \in \mathcal{N}_{\mathcal{Y}}$.

Theorem 2.17. Let $X=\left(X,+_{1}, \circ_{1},\|.\|_{1}, \mathbb{R}\right)$ and $Y=\left(Y,+_{2}, \circ_{2},\|.\|_{2}, \mathbb{R}\right)$ be normed hypervector spaces. The set $\mathcal{N}_{\mathcal{Y}}$, for $\mathcal{Y} \subseteq Y \times Y$, is a 2 -normed hyperset with the following 2-norm:

$$
\|f, g\|=\sup \left\{\|f(x), g(y)\|: x \in X, y \in Y,\|x\|_{1},\|y\|_{2} \leqslant 1\right\} \text {, }
$$

for $(f, g) \in \mathcal{N}_{\mathcal{Y}}$. Moreover, if $\mathcal{Y}$ is a symmetric 2 -normed set, then the set $\mathcal{N}_{\mathcal{Y}}$ is symmetric, too.

Remark 2.18. Note that the 2 -normed hypersets $\left(\mathcal{M}_{\mathcal{Y}},\|.,\|_{\mathcal{M}_{\mathcal{Y}}}\right)$ and $\left(\mathcal{N}_{\mathcal{Y}},\|., .\|_{\mathcal{N}_{\mathcal{Y}}}\right)$ have the following properties:

(i) $\mathcal{N}_{\mathcal{Y}} \subseteq \mathcal{M}_{\mathcal{Y}}$

(ii) $\|f, g\|_{\mathcal{M}_{\mathcal{Y}}} \leqslant\|f, g\|_{\mathcal{N}_{\mathcal{Y}}}$, for every $(f, g) \in \mathcal{N}_{\mathcal{Y}}$.

\section{REFERENCES}

1. S. Gähler, Linear 2-normierte Rume, Math. Nachr. 28(1964), 1-43. 1

2. Z. Lewandowska, Linear operators on generalized 2-normed spaces, Bull. Math. Soc. Sci. Math. Roumanie 42 (1999), 353-368. 1

3. Z. Lewandowska, On 2-normed sets, Glasnik Matematicki, 38(58) (2003), 99-110.

4. Z. Lewandowska, Bounded 2-linear operators on 2-normed sets, Glasnik Matematicki, 39(59) (2004), 303-314.

5. C. S. Lin, On strictly convex and strictly 2-convex 2-normed spaces, Math. Nachr, 148 (1990), 111-116. 1

6. A. Misiak, n-inner product spaces, Math. Nachr. 140 (1989), 299-319. 1

7. P. Raja, S. M. Vaezpour, Normed Hypervector Spaces, submitted. 1

8. P. Raja, S. M. Vaezpour, Strong Homomorphisms and Linear Functionals in Normed Hypervector Spaces, submitted. 1 
9. P. Raja, S. M. Vaezpour, Convexity in Normed Hypervector Spaces, to apear in Set-Valued Mathematics and Applications. 1

10. M. Scafati Tallini, A-ipermoduli e spazi ipervettoriali, Rivisita di Mat. Pura e Appl., Univ. Udine, 3 (1988) 39-48. 1

11. A. White, 2-Banach spaces, Math. Nachr. 42 (1969), 43-60. 1

1 Department of Mathematics and Computer Sciences, Amirkabir University of Technology, Hafez Ave., P. O. Box 15914, Tehran, Iran. Fax: +98-21-66497930. E-mail address: p_raja@cic.aut.ac.ir

2 Department of Mathematics and Computer Sciences, Amirkabir University of Technology, Hafez Ave., P. O. Box 15914, Tehran, Iran. Fax: +98-21-66497930.

E-mail address: vaez@aut.ac.ir 